\title{
The Heraclitean Universe: New Tools and Exotica from Transient Surveys of the Galaxy and Beyond
}

\author{
James M. Cordes ${ }^{* \dagger}$ \\ Cornell University, Ithaca, NY USA \\ E-mail: cordeseastro.cornell.edu
}

\begin{abstract}
The radio part of the electromagnetic spectrum holds great promise for major new discoveries of time-variable sources using new arrays being constructed and planned. This paper summarizes our knowledge of the variable radio sky and discusses likely yields and speculative possibilities for synoptic surveys. The role of plasma propagation effects in the interstellar and intergalactic media are discussed, including a definition of the pulse-broadening horizon for Galactic and cosmological sources of fast transients. Sources in the Galactic center and at cosmological distances — including pulsars orbiting Sgr A* and prompt counterparts to gamma-ray bursts — are particular examples of high-payoff targets of transient survey campaigns that require special attention to propagation effects in surveys. Once found, transient sources provide unique information about densities, turbulence and magnetic fields of the intervening media. Probing the ionized cosmic web is a particularly good example of where the medium may be the message and not the sources themselves. Synergies between radio and optical synoptic surveys are likely to be very fruitful because cross-statistical studies will provide strong filters for identifying exotic sources.
\end{abstract}

ISKAF2010 Science Meeting - ISKAF2010

June 10-14, 2010

Assen, the Netherlands

\footnotetext{
${ }^{*}$ Speaker.

${ }^{\dagger}$ This work was supported in part by the National Science Foundation through grant AST 0807151 to Cornell University and through cooperative agreements with Cornell to operate the Arecibo Observatory and to conduct the SKA Technology Development Project.
} 


\section{Introduction}

There is increasing interest in time variability of cosmic sources on all time scales. This interest is driven by two developments: (1) the recognition that, while there are prominent examples of transients extending to sub-ns durations, there have been no comprehensive surveys from the radio to the optical like those in gamma and X-rays; (2) the ability to do surveys for fast transients over large solid angles is now within our grasp owing to the evolution of digital processing and to the ability to build wide field of view telescopes. Much is known about radio transients [7] from both large single-dish telescopes (e.g. Arecibo, Parkes) and arrays. New array telescopes, including the EVLA, LOFAR, SKA precursors (ASKAP, MeerKAT) and the SKA itself will fulfill the goal of achieving a synoptic view of the radio sky on a large range of time scales that will complement those in other wavelength or energy bands [22].

In this paper I focus on radio transients that are astrophysical or astrobiological in origin, recognizing that bursts caused by cosmic ray showers from the Moon and Earth's atmosphere are also of great interest and will be discussed by Falke (these proceedings).

Fast radio transients can be defined loosely as those that are too fast to be sampled comprehensively with a raster-scan survey and, if sub-second or so, are likely associated with a coherent radiation process and with compact sources like neutron stars, black holes, exoplanets and extraterrestrial intelligence (ETI). Owing to the compactness of relevant sources and to the fastness itself, measured signals are subject to plasma propagation effects that are well known from pulsar observations, including pulse broadening and intensity scintillations from multipath propagation.

The following sections discuss the phase space for radio transients and key source classes along with propagation effects in the interstellar and intergalactic media, how comprehensive surveys can be conducted, and synergies with surveys at IR and optical wavelengths and at high energies are also discussed.

\section{Radio Transient Phase Space}

Nature can be profligate in producing radio photons and turn sources into bright, easilydetectable radio beacons. Under appropriate conditions, this occurs via coherent radiation processes that yield $N^{2}$ rather than $N$ times the radiation of a single particle in an $N$-particle system. In most sources, radio emission is not the dominant energy channel for dumping free energy but it is an extremely significant information channel. Examples include pulsars and radio pulses from ultra high-energy cosmic rays.

Giant pulses from radio pulsars are prototypes for fast transients along with solar bursts and stellar flares, while sources such as microquasars and gamma-ray burst (GRB) afterglows exemplify longer-duration transients. We may use these sources as initial guides for specifying blind-survey parameters. However, simple observational phase space arguments suggest that instantaneous coverage of a large fraction of the sky with appropriate sampling of the frequency-time plane will yield a rich variety of transient sources, including new classes of objects. Figure 1 shows the phase space of pulse width $W$ against flux density. Lines of constant brightness temperature are calculated 


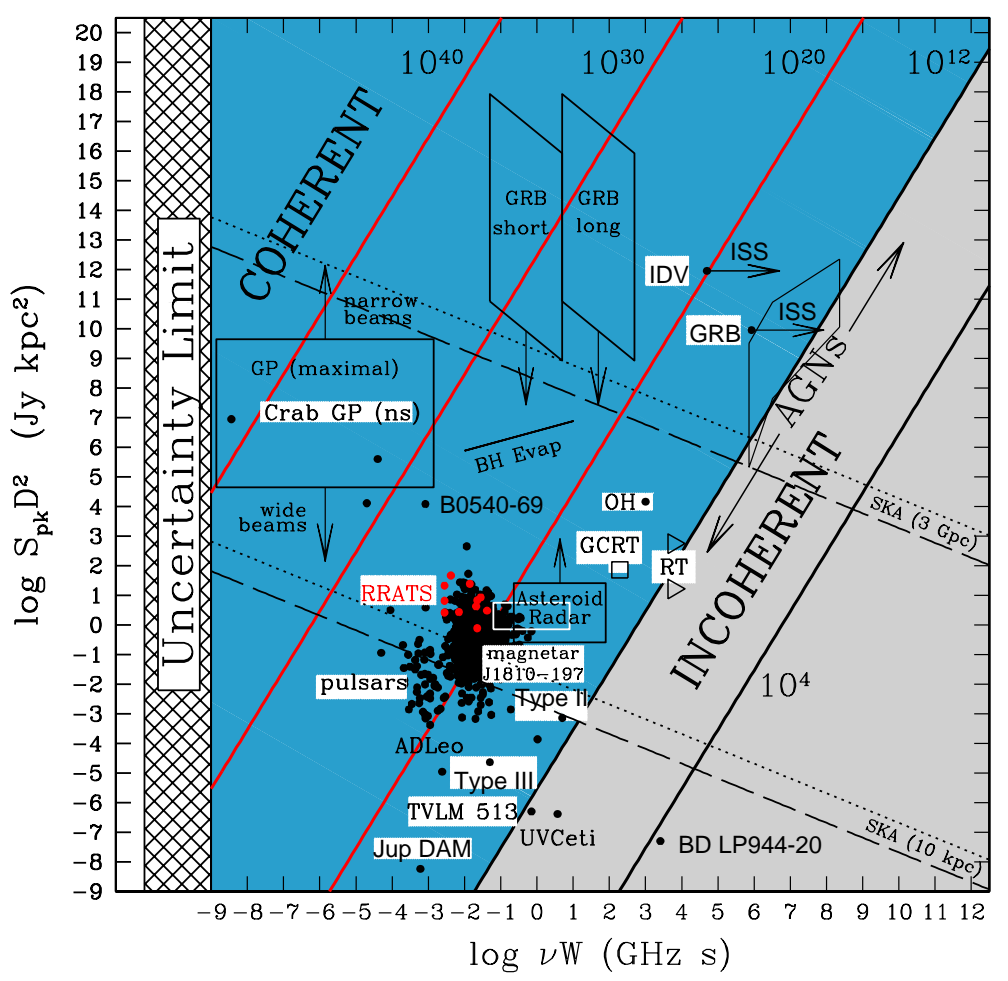

Figure 1: Time-luminosity phase space for known radio transients, a log-log plot of the product of peak flux $S_{\mathrm{pk}}$ in Jy and the square of the distance $D$ in kpc vs. the product of frequency $v$ in $\mathrm{GHz}$ and pulse width $W$ in s. The "uncertainty" limit on the left indicates that $v W \gtrsim 1$ as follows from the uncertainty principle. Lines of constant brightness temperature $T=S D^{2} / 2 k(v W)^{2}$ are shown, where $k$ is Boltzmann's constant. Points are shown for the 'nano-giant' pulses detected from the Crab [11], giant pulses detected from the Crab pulsar and a few millisecond pulsars, and single pulses from other pulsars. Points are shown for Jovian and solar bursts, flares from stars, brown dwarfs (e.g. TVLM 513-46546 [10]), OH masers, and AGNs. The regions labeled 'coherent' and 'incoherent' are separated by the canonical $10^{12} \mathrm{~K}$ limit from the inverse Compton effect that is relevant to incoherent synchrotron sources. Arrows pointing to the right for the GRB and intraday variable (IDV) points indicate that interstellar scintillation (ISS) implies smaller brightness temperatures than if characteristic variation times are used to estimate the brightness temperature. The growing number of recent discoveries of transients illustrates the fact that empty regions of the $v W-S_{\mathrm{pk}} D^{2}$ plane may be populated with sources not yet discovered. These include the "rotating radio transients" (RRATs) [17], the Galactic center transient source, GCRT J1745-3009 [13], the bright, bursting magnetar XTE J1810-197 [4], and transients (labelled "RT") associated with distant galaxies [3]. The rightward-directed triangles used to mark the two RT sources indicate that the transient durations are only known to be longer than $\sim 20$ min. Stellar bursts are included, such as recent observations of AD Leo [19] indicating $T_{b} \gtrsim 10^{18} \mathrm{~K}$. The figure also includes hypothetical transient sources and detection curves. Possible transients include maximal giantpulse emission from pulsars, prompt radio emission from GRBs, bursts from evaporating black holes, and radar signals used to track potentially impacting asteroids and comets in exosystems. Dashed lines indicate the detection threshold for the full SKA for sources at distance of $10 \mathrm{kpc}$ and $3 \mathrm{Gpc}$. Dotted lines correspond to a $10 \%$ SKA, comparable to the Arecibo telescope. At a given $v W$, a source must have luminosity above the line to be detectable. The curves assume optimal detection (matched filtering). 
assuming that $W$ is the light-travel time across the source,

$$
T_{b}=\frac{S}{2 k}\left(\frac{D}{v W}\right)^{2}=10^{20.5} \mathrm{~K} S_{\mathrm{mJy}}\left(\frac{D_{\mathrm{kpc}}}{v_{\mathrm{GHz}} W_{\mathrm{ms}}}\right)^{2},
$$

where $S_{\mathrm{mJy}}$ is the peak flux density (mJy) at frequency $v(\mathrm{GHz})$ and $D$ is the distance $(\mathrm{kpc})$. For some sources $W$ can be much smaller than the light travel time owing to relativistic compression.

It is useful to classify the wide range of known time scales in terms of how they are best sampled empirically.

Slow transients are defined as those with time scales longer than the time it takes to image the relevant region of the sky (e.g. the Galactic plane, the Galactic center, or the entire sky), either in a single pointing or as a mosaic or raster scan. Detection of such objects can be accomplished simply through repeated mapping of the sky and thus does not require special capabilities beyond those needed for imaging applications. Depending on survey speed, transients of days or more may be considered slow. GRBs are currently detected at $\gtrsim 100 \mu \mathrm{Jy}$ levels using the VLA at frequencies of 5 and $8 \mathrm{GHz}$ [9]. The full SKA could detect GRB afterglows (including orphan afterglows, e.g. [21]) at levels 10 to 100 times fainter than currently.

Fast transients, conversely, are those that would be missed in the time it takes to scan the sky, leading to incompleteness of the survey. Sub-second transients are linked to coherent radiation and, in many cases, to compact sources in extreme matter states. They also are affected by plasma propagation effects, such as dispersion and multipath scattering that can distort pulses and inhibit detection. By the same token, such transients are excellent probes of the intervening interplanetary, interstellar and intergalactic media. Fast transients require the same observing modes and postprocessing as for pulsars. The Crab pulsar is the most extreme known case in showing temporal structure down to $\sim 0.4 \mathrm{~ns}$ scales [11] and giant pulses that exceed 100 times the entire flux density of the Crab Nebula. Pulsars and giant-pulse emission are prototypes for coherent radiation that may originate from other high-energy objects in which collimated particle flows can drive plasma instabilities necessary for coherent radiation. Examples include prompt radio burst emission from GRB-type sources, perhaps even from gamma-ray quiet objects; flare stars, jovian-burst like radiation from planets, AGNs, and merging neutron stars at cosmological distances [12], some of which we designate in Figure 1. Photon reprocessing may limit the radio brightness of GRBs, so significant non-detections will constrain source conditions [16].

Using GRBs as a guide, it may be noted that the rate of GRB detections with gamma-ray instruments has relied more on instantaneous wide-field sky coverage than on sensitivity. The same statement holds for the detection of prompt optical emission at $m_{v}=9$ in at least one case [2]. For this reason, we suggest that use of subarray modes to increase the instantaneous sky coverage — at less than full sensitivity — will be productive in surveying the transient radio sky.

\section{Plasma Propagation Effects}

Intervening plasmas are the dominant causes of effects that modify radio emission from both steady and time-dependent sources. Of course plasma effects are accompanied by gravitatational lensing and redshift-dependent phenomena on cosmological lines of sight. 

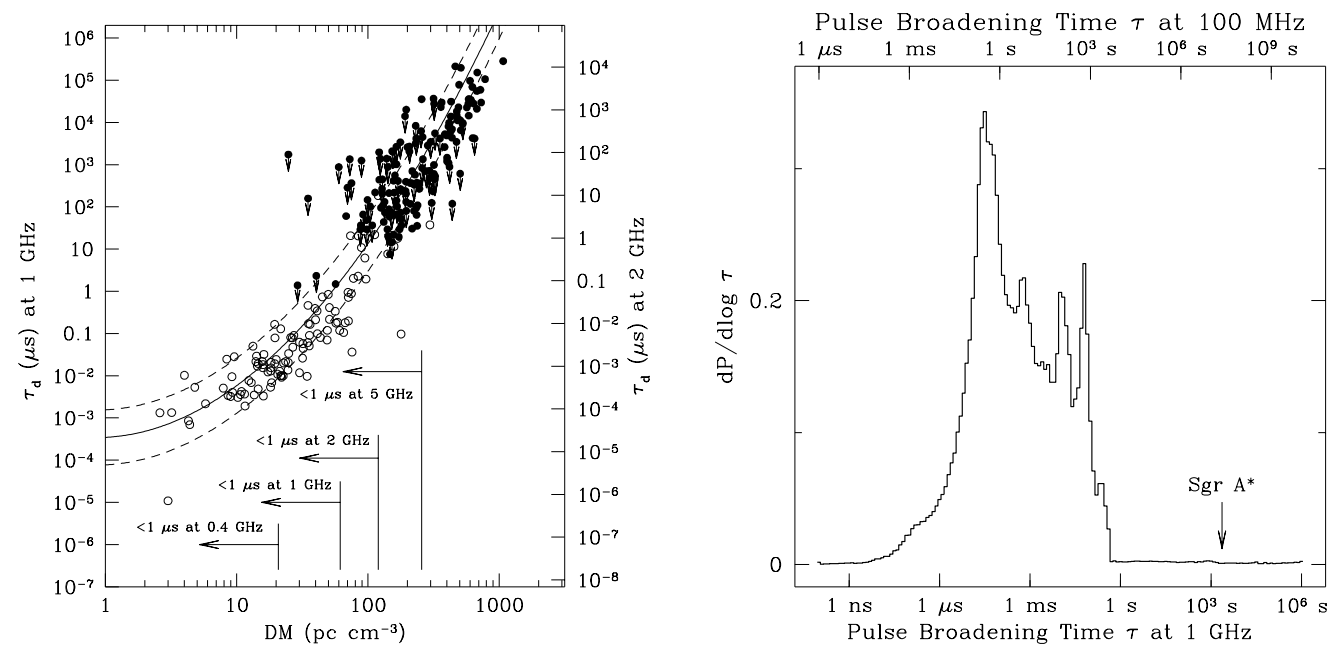

Figure 2: Left: Scatter plot of pulse broadening times vs dispersion measure for known pulsars. The solid line is a quadratic fit of $\log \tau_{d}$ to $\log \mathrm{DM}$ and the dashed lines are $\pm 1 \sigma$ deviations from the best fit. Downward going arrows are upper bounds. The vertical lines designate maximum DMs for which the scattering time is less than the indicated time scale. Right: Histogram of the pulse broadening time expected from sources distributed throughout the disk of the Milky Way, defined as a disk of radius $10 \mathrm{kpc}$ and thickness $1 \mathrm{kpc}$. The scattering measure and pulse broadening were calculated using the NE2001 model [6]. The bottom horizontal scale gives values for a radio frequency of $1 \mathrm{GHz}$ and the top axis for $100 \mathrm{MHz}$.

Dispersion: Fast transients have signal shapes modified by the strongly frequency-dependent travel time. When a pulse propagates dispersively, its arrival time varies with frequency as $\Delta t=$ $4.15 \mathrm{~ms} \mathrm{DM} v^{-2}$ for dispersion measure DM (the line-of-sight integral of electron density) in standard units $\left(\mathrm{pc} \mathrm{cm}^{-3}\right)$ and $v$ in GHz. Equivalently, the sweep rate in frequency is $\dot{v} \propto v^{3} \mathrm{DM}^{-1}$. If not compensated, a pulse measured across a finite bandwidth is smeared out. However, dedispersion techniques are well developed, allowing this deterministic effect to be largely mitigated. $A$ priori DM is not known, so trial values must be used from a set of plausible values. For Galactic sources, DM ranges between 2.4 and $\sim 1300 \mathrm{pc} \mathrm{cm}^{-3}$ among known pulsars. The NE2001 model for free electrons in the Galaxy [6] predicts dispersion measures up to about $3400 \mathrm{pc} \mathrm{cm}^{-3}$ when looking through the Galactic center region. However, much larger values will obtain for lines of sight that pierce dense HII regions.

Extragalactic sources will show contributions to DM from foreground Galactic electrons as well as from a host or intervening galaxy (if relevant) and from the intergalactic medium (IGM). A fiducial value for intergalactic dispersion is

$$
\mathrm{DM}_{\mathrm{igm}}=\mathrm{cH}_{0}^{-1} \mathrm{n}_{\mathrm{e} 0} \approx 10^{3} \mathrm{pc} \mathrm{cm}^{-3},
$$

where $n_{e 0}$ is the electron density associated with the baryonic fraction of the total closure density. Scattering and Scintillation: Multipath propagation along Galactic lines of sight occurs because there is microstructure in the free electrons down to scales $\sim 10^{3} \mathrm{~km}$ (e.g. [20]), from which radio waves diffract and refract. Relevant effects include angular broadening ("seeing”), pulse broadening, and intensity scintillations. 
Measured pulse broadening on Galactic lines of sight is as large as $\sim 1 \mathrm{~s}$ but scales very strongly with frequency, $\tau_{d} \propto v^{-4}$. Figure 2 shows the distribution of $\tau_{d}$ expected using the electron density model NE2001 [6]. On lines of sight to the Galactic center, $\tau_{d}$ is thousands of seconds at $1 \mathrm{GHz}$ and prohibits the detection of pulsars around $\mathrm{Sgr} \mathrm{A}^{*}$ at conventional frequencies used in pulsar searches. Searches for fast transients from the Galactic center region therefore must be done at high frequencies $\gtrsim 10 \mathrm{GHz}$, where $\tau_{d}$ is $\sim 10^{4}$-times smaller.

Diffractive scintillation (DISS) results from interference between scattered wavefronts and produces structure in both time and frequency with representative scales of $100 \mathrm{~s}$ and $0.1 \mathrm{MHz}$, but with ranges in each of many orders of magnitude. Refractive scintillation (RISS) is caused by focusing and defocusing of radiation from scales much larger than those responsible for DISS in the strong-scattering regime. RISS is broadband, $\Delta v / v \sim 1$, and has time scales of hours and longer, depending on the line of sight. RISS from Galactic plasma appears to underly the "intraday variability" (IDV) of some active galactic nuclei.

Broadening of pulses from extragalactic sources by intergalactic or extragalactic plasma has not yet been measured definitively. Propagation through a face-on intervening galaxy like the Milky Way would scatter radiation into $\sim 1$ mas at $1 \mathrm{GHz}$ and broaden a pulse by a fiducial broadening time

$$
\Delta t_{\text {face on }}=\frac{\theta_{s}^{2}}{2 H_{0}}=5 \theta_{s, \text { mas }}^{2} \text { sec. }
$$

Pulse broadening from a host galaxy is deleveraged from this value by a geometrical factor $\sim H / D$ where $H$ is the path length through the host galaxy and $D$ is the distance. Pulse broadening could be much larger, however, from edge-on and starburst galaxies and the IGM, if turbulent like the interstellar medium, could also make a sizable contribution. Detection of pulses from cosmological sources would give a very powerful tool for probing the IGM as would relatively nearby sources in local-group galaxies.

\subsection{The Pulse Broadening Horizon}

Pulses of given intrinsic width $W$ can be detected only from those sources near enough to not be broadened significantly. From Figure 2 we can define the Galactic horizon in terms of DM by requiring that $\tau_{d} \lesssim W$. For $1 \mathrm{~ms}$ pulses, the horizon is about $5 \mathrm{kpc}$ at $1 \mathrm{GHz}$ while $1 \mu \mathrm{s}$ pulses can be seen to only $2.4 \mathrm{kpc}$ at $1 \mathrm{GHz}$. These values apply only to sources within the Galactic disk. Looking perpendicular to the disk, the seeing distance "breaks out" if it is more than about $1 \mathrm{kpc}$. At low frequencies, e.g. $100 \mathrm{MHz}$, a $1 \mu \mathrm{s}$ pulse can be seen only to about $100 \mathrm{pc}$.

The cosmological horizon can be calculated form the variation of DM with redshift $z$ assuming that the IGM is completely ionized and that the relationship of pulse broadening time to DM is the same as for Galactic sources; this is at best a very crude approach. We find that a $1 \mathrm{~ms}$ pulse can be detected to $z \approx 0.2$ and the broadening is $\tau_{d} \sim 100 \mathrm{~ms}$ from sources at $z=1$.

\section{How Bright Can Fast Transients Be?: Giant and Hyper-giant Pulses}

We already know that giant pulses from certain pulsars, like the Crab, are emitted frequently enough to be detectable at plausible rates out to $1.5 \mathrm{Mpc}$ with Arecibo. This number results from 
scaling $0.43 \mathrm{GHz}$ pulses of amplitude $S_{\mathrm{pk}} \sim 150 \mathrm{kJy}$ that are pulse-broadened to $\sim 100 \mu$ s duration. Such pulses occur from the Crab at a rate $\sim 1 \mathrm{hr}^{-1}$. They correspond to a pseudo-luminosity $S_{\mathrm{pk}} D^{2} \sim 10^{5.8} \mathrm{Jy} \mathrm{kpc}^{2}$. Surely there are more luminous, giant-pulse-emitting pulsars that are detectable even further.

The case can be made that there are other burst sources that can tap larger sources of free energy than are available in pulsars like the Crab. These alternatives may include:

- Hyperfast rotators: Pulsars born near the break-up limit ( $\sim 1 \mathrm{~ms})$ with canonical or magnetarlike magnetic fields ( $10^{12}$ to $10^{15}$ Gauss) can rapidly dump their rotational energy (using a moment of inertia of $10^{45} \mathrm{gm} \mathrm{cm}^{2}$ ),

$$
\frac{1}{2} I \Omega^{2}=2 \pi^{2} I P^{-2} \sim 10^{51.3} \operatorname{erg} I_{45} P_{\mathrm{ms}}^{-2},
$$

which is comparable to the non-neutrino energy released in a supernova. This energy will be released in a short amount of time as the pulsar rapidly spins down but along the way can drive giant-pulse emission much larger than seen from the Crab, e.g. by a factor of one million, e.g $S_{\mathrm{pk}} D^{2} \sim 10^{12} \mathrm{Jy} \mathrm{kpc}^{2}$.

- Prompt GRB Counterparts: Neutron stars may reactivate their magnetospheres when they merge, with orbital motion substituting for spin in the generation of voltage drops that accelerate particles and create electron-positron pairs. This kind of system is a strong candidate for short-duration gamma-ray bursts. The energy involved is similar to or exceeds that for extreme giant pulse-emitting objects. The GRB peak luminosity is fiducially $L_{\gamma}=10^{51} L_{\gamma, 51} \mathrm{erg} \mathrm{s}^{-1}$. We assume that the true radio luminosity is some multiple $\varepsilon_{r}$ of this: $L_{r}=\varepsilon_{r} L_{\gamma}$. Remarkably, over many kinds of astrophysical objects (stars, pulsars, AGNs), we find $\varepsilon_{r}$ ranging from about $10^{-8}$ (Crab pulsar) to $10^{-3}$ (blazars). Here we use a fiducial value $\varepsilon_{r}=10^{-5} \varepsilon_{r,-5}$ and a radio emission bandwidth $\Delta v_{r}=1 \mathrm{GHz} \Delta v_{r, \mathrm{GHz}}$. We then

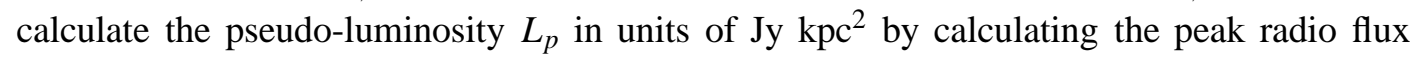
from the GRB assuming (only fiducially) isotropic emission. This gives a stupendously large pseudo-luminosity,

$$
L_{p}=10^{15.9} \mathrm{Jy} \mathrm{kpc}^{2}\left(\frac{\varepsilon_{r,-5} L_{\gamma, 51}}{\Delta v_{r, \mathrm{GHz}}}\right)
$$

corresponding to a peak flux density

$$
S_{\mathrm{pk}}=10^{2.9} \mathrm{Jy}\left(\frac{\varepsilon_{r,-5} L_{\gamma, 51}}{\Delta v_{r, \mathrm{GHz}}}\right)\left(\frac{3 \mathrm{Gpc}}{d_{L}}\right)^{2} .
$$

Beaming may influence the radio luminosity estimate as it does the $\gamma$-ray luminosity.

A 30 Jy pulse at $1.4 \mathrm{GHz}$ has been detected that has $\mathrm{DM} \sim 375 \mathrm{pc} \mathrm{cm}^{-3}$ from a direction toward which the general interstellar media in the Galaxy and in the Small Magellanic Cloud cannot account for the electrons [15]. The authors argue for a $500 \mathrm{Mpc}$ distance based on the DM from the Milky Way and from the IGM. This corresponds to $S_{\mathrm{pk}} D^{2} \sim 10^{13} \mathrm{Jy} \mathrm{kpc}^{2}$, much larger than any known Galactic source but comparable to the maximal giant-pulse emission discussed above while being somewhat less than our estimate for prompt GRB emission (with admittedly much latitude on scaling parameters). 


\section{Radio Synoptic Surveys}

Surveys of time-variable sources need to take into account event rates and durations as well as sensitivity requirements. Transients cover a very wide dynamic range in time scale. Slow transients may be sampled by raster scanning, covering a total solid angle $\Omega_{s}$ in a time $T_{\mathrm{s}}$ and then repeating the scan. The dwell time per sky position is $\tau=\left(\Omega_{i} / \Omega_{s}\right) T_{s}$, where $\Omega_{i}$ is the instantaneous FoV. Slow transients are also those for which pulse broadening from plasma dispersion and multipath propagation are unimportant. Fast transients are those that cannot be well sampled through raster-scan imaging surveys, unless they are very frequent and there is tolerance for low survey completeness. Transients shorter than about one day qualify as fast if an all-sky survey is contemplated. Rare, fast transients are better sampled through staring observations of large solid angles. ${ }^{1}$

\subsection{Trading Field of View and Sensitivity}

If there are hyper-strong events (such as coherent radio pulses from GRBs), a wide FoV system is necessary for a reasonable detection rate, but modest collecting area may suffice for detection. However, rare weak events require high sensitivity as well as wide FoV. Field of view expansion of arrays of single reflectors allows probing of this region of phase space as do aperture arrays, such as LOFAR and those proposed for low frequencies with the SKA. But it is also true that wide FoV may be achieved at moderate sensitivity through the use of subarrays. Since the low-event-rate radio sky is largely unexplored, we should entertain the possibility of using the subarray approach as well as develop FoV expansion approaches.

\subsection{The Synoptic Cycle: An Example of Multiple Commensal Surveys}

Though processing requirements are challenging, there is no fundamental reason why commensal observations in a synoptic survey mode with the SKA (and precursor instruments) cannot provide a massive survey of HI in galaxies, large numbers of Faraday rotation measures, a fullGalactic census of pulsars, and unprecedented characterization of the transient radio sky that, together, are the basis for profound progress in fundamental physics, cosmology, astrophysics and astrobiology as well as providing discoveries of entirely new sources and phenomena.

The observing cycle for a given survey might consist of a raster scan of a region of sky $\Omega_{\mathrm{s}}$ in a time $T_{\mathrm{s}}$, yielding a dwell time per sky position $\tau=T_{\mathrm{s}} \Omega_{i} / \Omega_{s}$. In practice this rate may be achieved by continuous scanning or by dwelling on each of a predefined grid of positions [8]. This cycle can be repeated many times, allowing detection of a growing number of transient sources, both periodic ones like pulsars and magnetars, and one-shot objects like GRBs. At the same time, signal-to-noise ratio is built up for steady sources, such as HI in galaxies, continuum sources for AGN surveys and Faraday rotation measurements. Because multiple surveys are potentially doable, and each

\footnotetext{
${ }^{1}$ In terms of number of detected events, it is equivalent to cover a large solid angle through raster scanning and small duty cycle per sky position, or to cover a smaller solid angle with continuous time coverage. If the goal is to characterize the angular distribution of the transient population, then raster scanning is appropriate. However, if the event rate of particular sources is desired or if the source population is restricted to a small solid angle (e.g. the Galactic center region), then staring observations are needed.
} 
is demanding on total telescope time, all effort should be made to do the surveys simultaneously (commensally).

To accomplish multiple surveys, a hierarchical approach to scanning rates (or cadences) is probably needed. A fast rate is appropriate for the extragalactic sky in order to sample GRB afterglows while also building S/N on HI galaxies. A slower rate is needed for the Galactic disk to provide adequate time series durations for pulsar surveys. Staring observations of the Galactic center source will allow deep pulsar and transient surveys from the star cluster. Finally, guest-investigator experimental or one-time observations need to be accomodated along with target-of-opportunity observations as they arise.

An example scenario includes both fast and slow scanning observations and staring observations. This example sums to about 10 days per cycle, which would be repeated as needed:

1. Fast scan of the extragalactic sky: large-scale galaxy HI survey, Faraday rotation survey, AGN survey, transients and high-latitude pulsars (millisecond pulsars and those in relativistic binaries with NS or blackhole companions):

(a) "Full sky" survey ( $80 \%$ of $4 \pi$ ) using a $1 \mathrm{deg}^{2}$ FoV single pixel system

(b) $T_{\mathrm{s}}=5$ days to cover one scan of the sky

(c) $\tau \approx 10 \mathrm{~s}$ dwell time per sky position

(d) $S_{\min } \approx\left(g_{\theta} f_{\mathrm{c}}\right)^{-1} 15 \mu \mathrm{Jy}$ at $10 \sigma$ where $f_{\mathrm{c}}$ is the fraction of a full SKA available and $g_{\theta} \leq 1$ is the gain relative to the on-axis gain

(e) Field-of-view expansion through multiple feed clusters or phased-array feeds will increase the sensitivity for fixed $T_{\mathrm{s}}$; aperture arrays would also provide an increase. A thirty-beam phased-array feed, for example, would yield $300 \mathrm{~s}$ dwell time

(f) Subarrays will reduce the sensitivity but can cover more instantaneous solid angle and reduce $T_{\mathrm{s}}$.

(g) Commensal observations require appropriate real-time, backend processing systems that will cost a significant fraction of the overall cost of a synoptic telescope.

2. Slower scan or staring observations on deep extragalactic fields e.g. 1 day

3. Slower scan for the Galactic plane: pulsars, masers, transients, ETI sources, etc. e.g. 1 day scan of the inner Galaxy (180 deg in longitude) in a \pm 1 deg swath in Galactic latitude, yielding $240 \mathrm{~s}$ per pointing

(a) A minimum contiguous dwell time is needed for pulsar surveys that use Fast Folding Algorithms or Fourier transforms of a contiguous time series combined with harmonic summing (100 to $1000 \mathrm{~s}$ typical); single-pulse searches do not place strong requirements on contiguous blocks.

(b) Pulsar timing: frequent re-observations are needed for long-term monitoring; a 10day cadence is acceptable, so that timing measurements can be obtained as part of the survey.

4. Staring observations: e.g. $12 \mathrm{hr}$ on the Galactic center to detect pulsars and transients.

5. Break out for targeted observations by individual investigators: $10 \%$ of the time? 
6. Break out for targets of opportunity: e.g. GRB triggers, blazar observations, etc. $5 \%$ of the time?

\section{Calibration allowance}

Additional comments on this scenario are as follows:

1. The distinction between "survey" and "follow up" observations is fuzzy. For example, timing observations of known and new pulsars can be obtained using the same survey scans that yield detections of new sources.

2. HI detection of galaxies at $z \sim 1$ requires many hours of integration time, which would build up slowly unless there is field-of-view expansion.

3. Pulsar surveys can accumulate $\mathrm{S} / \mathrm{N}$ through incoherent summing of power spectra from noncontiguous data segments, with due allowance for acceleration of the source or observer. Coherent sums across multiple days are probably too demanding computationally, but with requirements that depend on the cadence.

4. Diffractive and refractive interstellar scintillations (DISS and RISS) will modulate compact sources to varying degrees and with a wide range of correlation times. To optimize detection, multiple passes on the same sky position should be uncorrelated with respect to DISS and RISS [5].

\section{Multi-wavelength and Multi-Messenger Synergies}

Cross-wavelength studies of transient phenomena are the most powerful pathway for making new discoveries and understanding source classes. As is well established, GRBs have triggered observations across the entire spectrum and also signal exploration in gravitational wave data. Radio events, including rotational glitches in neutron stars, will play an increasing role as triggers. The forthcoming era of large synoptic survey telescopes will provide the data needed for data mining and source classification. For example the science case for the Large Synoptic Survey Telescope [1] at optical wavelengths identifies variable stars, novae, supernovae of various kinds and orphan afterglows as primary source classes along with gravitational lensing modulations. These transients are mostly on times scales on one day or longer. In Venn-diagram space, there will be only partial overlap between radio and optical/IR (OIR) source classes. In fact, any joint hits between radio and OIR samples are likely to be extremely interesting. Inclusion of high-energy events will make the cross wavelength results even richer. Finally, merger events that likely produce short-hard GRBs and certainly produce gravitational wave events detectable with advanced LIGO, VIRGO or future GW detectors, may also generate coherent radio bursts. If radio bursts are associated with the interactions of the magnetospheres, they may precede the high-energy bursts and gravitational wave events, even after taking into account dispersion delays through the IGM.

\section{Acknowledgments}

I thank R. Bhat, G. Bower, J. Deneva, R. Ekers, M. Kramer, S. Kulkarni, J. Lazio, D. Lorimer, A. Lyne, J-P. Macquart, M. McLaughlin, R. Schilizzi, R. Shannon, and D. Werthimer for useful discussions. 


\section{References}

[1] Abell, P. A. et al. 2009, LSST Science Book, Version 2.0, ArXiv e-print, 0912.0201

[2] C. Akerlof et al., Observation of Contemporaneous Optical Radiation from a Gamma-ray Burst, Nature, 398, (400) 1999

[3] G. C. Bower et al, Submillijansky Transients in Archival Radio Observations, ApJ, 666 (346) 2007

[4] F. Camilo et al., Transient Pulsed Radio Emission from a Magnetar, 2006, Nature, 442 (892) 2006

[5] J. M. Cordes \& T. J. W. Lazio, Interstellar scattering effects on the detection of narrow-band signals, ApJ, 376 (123) 1991

[6] J. M. Cordes \& T. J. W. Lazio, NE2001. I. A New Model for the Galactic Distribution of Free Electrons and its Fluctuations [astro-ph/0207156]

[7] J. M. Cordes, T. J. W. Lazio \& M. A. McLaughlin, The Dynamic Radio Sky, New Astronomy Review, 48 (1459) 2004

[8] J. M. Cordes, The Square Kilometer Array as a Radio Synoptic Survey Telescope: Widefield Surveys for Transients, Pulsars and ETI, SKA Memo 97, http://www.skatelescope.org, 2007

[9] D. A. Frail et al., An Energetic Afterglow from a Distant Explosion, ApJL, 646 (99) 2006

[10] G. Hallinan, et al., Periodic Bursts of Coherent Radio Emission from an Ultracool Dwarf, ApJL, 663 (25) 2007

[11] T. H. Hankins \& J. A. Eilek, Radio Emission Signatures in the Crab Pulsar, ApJL, 670 (693) 2007

[12] B. M. S. Hansen \& M. Lyutikov, Radio and X-ray Signatures of Merging Neutron Stars, MNRAS, 322 (695) 2001

[13] S. D. Hyman et al., A New Radio Detection of the Transient Bursting Source GCRT J1745-3009, ApJ, 639 (348) 2006

[14] M. Kramer et al.. A Periodically Active Pulsar Giving Insight into Magnetospheric Physics, Science, 312 (549) 2006

[15] D. R. Lorimer et al., A Bright Millisecond Radio Burst of Extragalactic Origin, Science, 318 (777) 2007

[16] J.-P. Macquart, On the Detectability of Prompt Coherent Gamma-Ray Burst Radio Emission, ApJL, 658 (1) 2007

[17] M. A. McLaughlin, et al., Transient Radio Bursts from Rotating Neutron Stars, Nature, 439, (817) 2006

[18] Osten, R. A. et al. 2010 The Mouse that Roared: A Superflare from the dMe Flare Star EV Lac detected by Swift and Konus-Wind, ArXiv e-print 1007.5300

[19] Osten, R. A. and Bastian, T. S. Ultrahigh Time Resolution Observations of Radio Bursts on AD Leonis, ApJ, 674 (1078) 2008

[20] B. J. Rickett, Radio Propagation Through the Turbulent Interstellar Plasma, ARAA, 28 (561) 1990

[21] Soderberg, A. M. et al. 2010, A Relativistic Type Ibc Supernova without a Detected $\gamma$-ray Burst, Nature, 463, 513

[22] Wozniak, P. R. et al. Explosive Transients in the Distant Universe, in Astro2010: The Astronomy and Astrophysics Decadal Survey, 2009astro2010S.326W, ArXiv Astrophysics e-print, 